Proceedings

\title{
Piezoelectric Actuators for In-Liquid Particle Manipulation in Microfluidic Applications ${ }^{+}$
}

\author{
Marco Demori *, Marco Baù, Simone Dalola, Marco Ferrari and Vittorio Ferrari \\ Department of Information Engineering, University of Brescia, 25123 Brescia, Italy; marco.bau@unibs.it (M.B.); \\ simone.dalola@unibs.it (S.D.); marco.ferrari@unibs.it (M.F.); vittorio.ferrari@unibs.it (V.F.) \\ * Correspondence: marco.demori@unibs.it \\ + Presented at the Eurosensors 2017 Conference, Paris, France, 3-6 September 2017.
}

Published: 8 August 2017

\begin{abstract}
The possibility to generate acoustic modes based on FPWs (Flexural Plate Waves) in a generic non-piezoelectric substrate for microfludic applications by means of piezoelectric actuators has been explored and described in this paper. The FPW acoustic modes are generated by means of actuators made of Lead Zirconate Titanate (PZT) layers with InterDigital Transducers (IDTs) screen-printed on alumina $\left(\mathrm{Al}_{2} \mathrm{O}_{3}\right)$ substrate. The experimental results show that, by exciting the resonances of the actuators, circular vortex rotations are obtained in a fluid drop placed on the substrate between the IDTs. Micrometric particles dispersed in the drop allow to demonstrate that standing waves can be generated in the liquid obtaining particle accumulation along circular lines. These results suggest the possibility to employ the proposed actuators for fluid mixing and controlled positioning of dispersed particles.
\end{abstract}

Keywords: acoustic wave; in-liquid particle manipulation; FPWs flexural plate waves; piezoelectric actuators

\section{Introduction}

Controllable and accurate fluid and particle manipulation in microfluidic devices represents an important tool for many applications in biological, chemical and physical research. The forces generated by the interaction between acoustic waves and fluids can be used to actuate fluids and suspended particles, such as cells, at small scale with micrometric resolution. The ability of SAWs (Surface Acoustic Waves) to generate motion of liquid drops or vortex rotation of the fluid in a drop was demonstrated for fluid steering and mixing applications [1,2]. Standing acoustic waves can be generated by a pair of opposite actuators obtaining pressure nodes in fluids. The possibility to operate controlled positioning of particles dispersed in liquid and accumulate them at the positions of pressure nodes was shown in [1-4].

In this paper, the possibility to generate acoustic waves based on FPWs (Flexural Plate Waves) [5] in a generic non-piezoelectric substrate to manipulate liquid and dispersed particles has been explored. The use of piezoelectric films fabricated by the screen-printing technique for the generation of the FPWs on non-piezoelectric substrate is proposed. The aim is that fluid and particle manipulations by acoustic waves can be advantageously obtained on generic non-piezoelectric substrates, including transparent substrates typically adopted for the fabrication of microfluidic devices.

\section{Device Description}

A device with two Lead Zirconate Titanate (PZT) piezoelectric actuators with IDT electrodes fabricated by the screen-printing technique for the generation of acoustic waves in alumina substrate 
is proposed, as shown in Figure 1. The alumina substrate has a circular shape with 25-mm diameter and a thickness of $635 \mu \mathrm{m}$; the actuators are located diametrically opposed.

The actuators consist of the PdAg bottom electrode, the PZT layer, and the PdAg electrodes of the IDT printed on top. IDTs have both finger width and spacing of $350 \mu \mathrm{m}$. The thickness $t_{p}$ of the PZT layer is about $100 \mu \mathrm{m}$. The PZT films were poled at $4 \mathrm{MV} / \mathrm{m}$ along the thickness using the IDT pair and the bottom electrode as the two armatures. During the excitation the bottom electrode is left floating and a sinusoidal voltage is applied between the two IDT fingers. Considering that the finger width and spacing are larger than the PZT layer thickness and the existence of the bottom conductive layer, the electric field in the PZT under the electrodes is predominantly oriented along the thickness. The applied excitation generates thus an alternate deformation of the PZT-layer thickness by exploiting mainly the $d_{33}$ mode, thus acting as actuator for the generation of the acoustic waves in the substrate [1]. The adopted PZT thickness $t_{P}$ sets the thickness resonances of the actuators in the range of few megahertz [6]. Fluid samples can be placed on the alumina substrate between the actuators to undergo the actuation resulting from the combination of the acoustic wave-fronts.

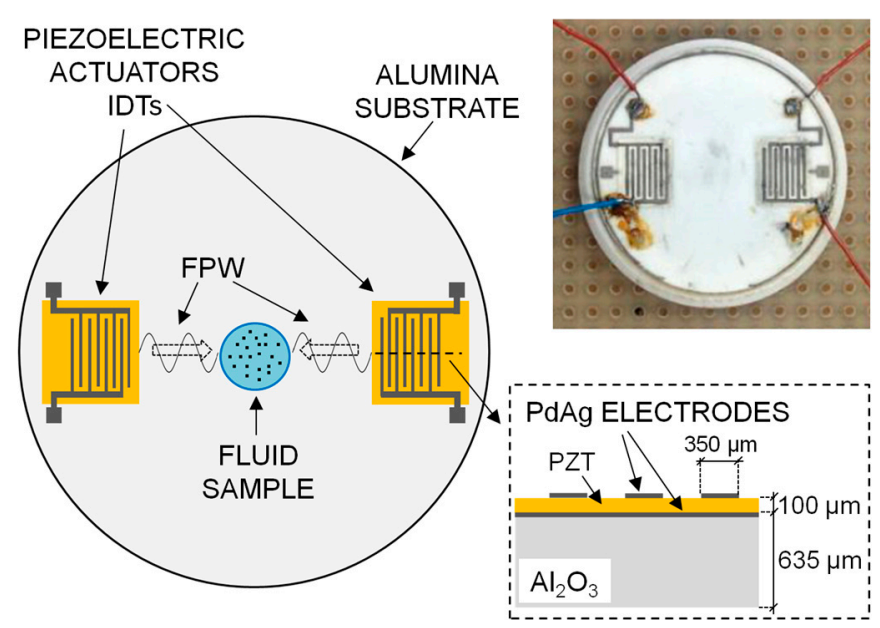

Figure 1. Sketch and picture of the fabricated device with a cross section of the actuators. Poling was done along the thickness using the bottom electrode.

\section{Experimental Results}

The actuators have been experimentally characterized in order to extract the frequencies where the maximum actuation effectiveness is expected. Figure $2 \mathrm{a}$ shows the admittance measured between the IDT electrodes evidencing resonances at about $f_{1}=6.7 \mathrm{MHz}, f_{2}=8.2 \mathrm{MHz}$ and $f_{3}=12$ $\mathrm{MHz}$. The expected wavelengths of the acoustic waves in the substrate at these frequencies can be calculated starting from the thickness $t_{A}=635 \mu \mathrm{m}$, the Poisson ratio $v=0.21$ and the shear wave velocity $v s=5774 \mathrm{~m} / \mathrm{s}$ for the adopted alumina substrate. Assuming that the generated acoustic waves have wavelengths much smaller than the substrate diameter, the Lamb waves theory for free isotropic elastic plates can be applied [7]. The propagation velocities and the wavelengths of the generated symmetric $S_{n}$ and antisymmetric $A_{n}$ modes can be derived from the tabulated Lamb dispersion curves for the specific value of $v$, where the waves velocity of the different modes is reported as a function of the frequency-thickness product. The derived wavelengths of the lower modes $\lambda\left(\mathrm{A}_{0}\right)$ and $\lambda\left(\mathrm{S}_{0}\right)$, which correspond to the shorter wavelengths generated in the substrate at the actuator resonances $f_{1}, f_{2}$ and $f_{3}$, are reported in Table 1 . The propagation of FPWs in the substrate is expected at these frequencies, corresponding to acoustic wavelengths comparable or larger than the substrate thickness $t_{A}$.

Figure $2 b$ shows the schematic diagram of the tailored electronic interface developed to drive the piezoelectric actuators. The excitation signals are generated by a programmable DDS (Direct Digital Synthesizer) that allows to excite the IDTs with two independent channels for which both the common excitation frequency and the phase difference between the channels can be set. 
Table 1. Calculated wavelengths of the lower acoustic modes generated in the substrate.

\begin{tabular}{ccc}
\hline Frequency & $\lambda\left(\mathbf{A}_{\mathbf{0}}\right)$ & $\lambda\left(\mathbf{S}_{\mathbf{0}}\right)$ \\
\hline$f_{1}=6.7 \mathrm{MHz}$ & $0.74 \mathrm{~mm}$ & $1.16 \mathrm{~mm}$ \\
$f_{2}=8.2 \mathrm{MHz}$ & $0.6 \mathrm{~mm}$ & $0.77 \mathrm{~mm}$ \\
$f_{3}=12 \mathrm{MHz}$ & $0.44 \mathrm{~mm}$ & $0.45 \mathrm{~mm}$ \\
\hline
\end{tabular}

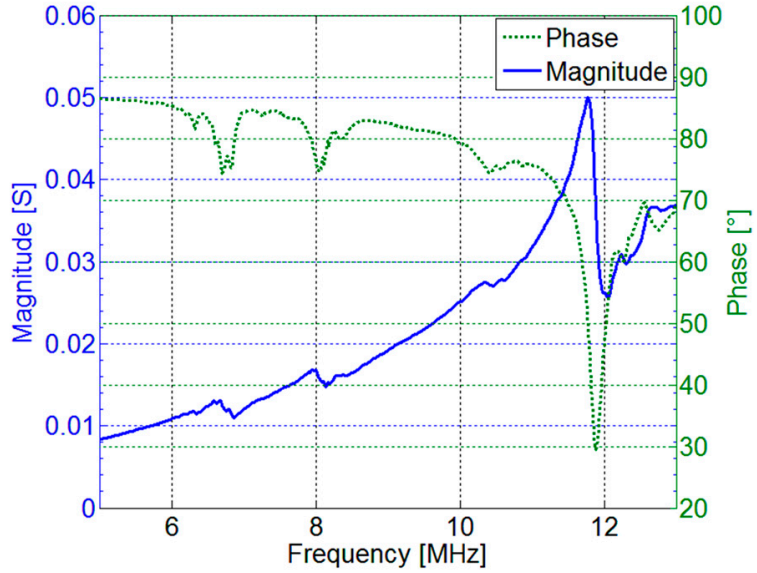

(a)

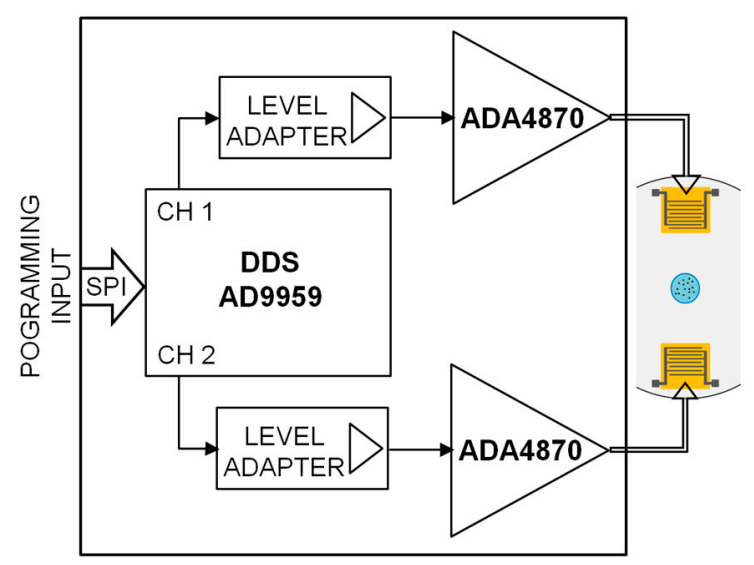

(b)

Figure 2. (a) Measured admittance (magnitude and phase) between the IDT electrodes as a function of frequency; (b) Block diagram of the tailored electronic interface designed to excite the actuators with sinusoidal signals with peak-to-peak amplitude and frequency of up to $20 \mathrm{~V}$ and $15 \mathrm{MHz}$, respectively.

Preliminary tests have been conducted to verify the ability of the generated acoustic waves to manipulate fluid drops and particles. The device has been placed under the microscope and a water drop with dispersed light-reflective microspheres has been placed on the alumina substrate between the actuators to acquire by a cam the generated fluid and particle motion, as shown in Figure 3a. The comparison between Figure $3 b$ and $3 c$ shows that circular vortex rotations have been obtained in the drop by exciting a single actuator with a sinusoidal signal at frequency of $12 \mathrm{MHz}$. By exciting both the actuators at frequency of $8.2 \mathrm{MHz}$ and phase difference of $0^{\circ}$, particle accumulation along circular lines caused by the standing waves generated in the drop has been obtained, as shown in Figure 3d.

\section{Conclusions}

The possibility to generate acoustic waves for fluid and particle manipulation in non-piezoelectric substrates using PZT film actuators fabricated by the screen-printing technique has been demonstrated. A device with two PZT actuators on alumina substrate has been realized. Experimental results show that both vortex rotation and accumulation on circular lines of micrometric particles dispersed in a water drop have been obtained by exciting the actuators at their resonant frequencies, demonstrating the actuation by means of acoustic waves. These results show the ability of the proposed PZT actuators to generate acoustic waves for fluid mixing and are promising for promoting and controlling the aggregation of particles dispersed in a liquid, such as cells, in microfluidic devices. 


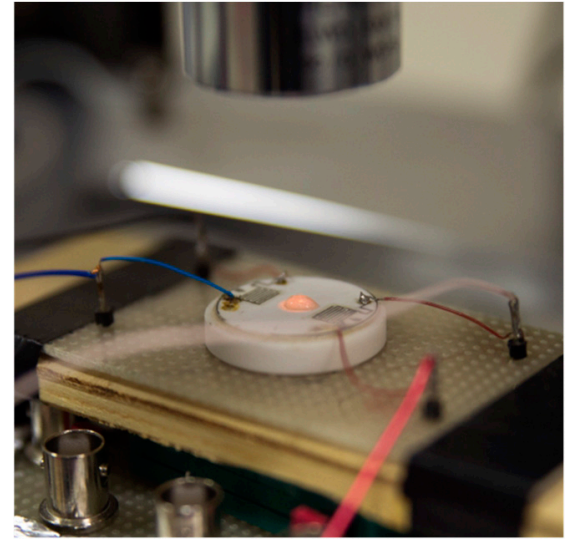

(a)

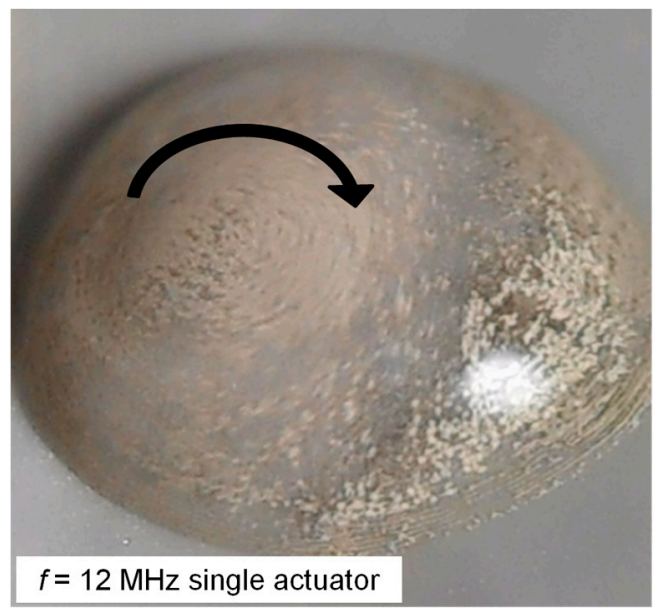

(c)

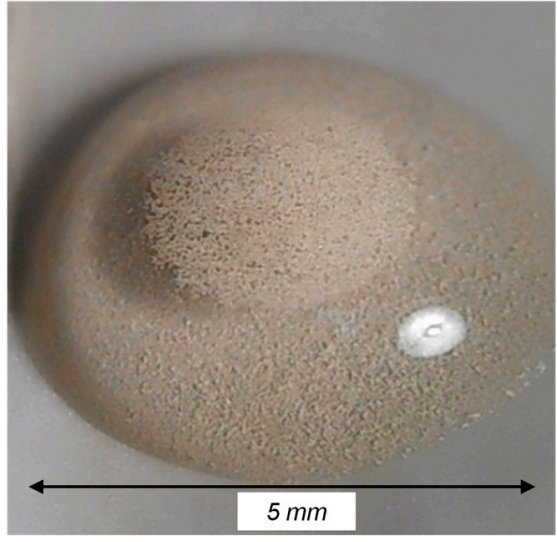

(b)

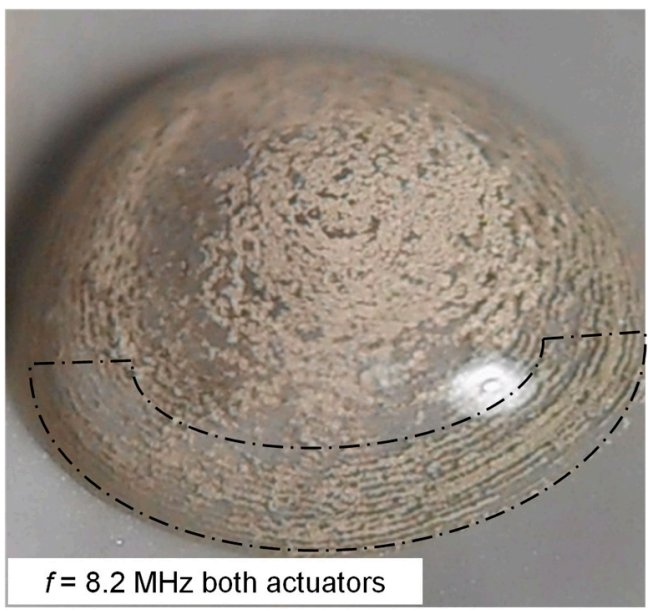

(d)

Figure 3. (a) Experimental setup showing the device placed under the microscope; Pictures of a water drop with dispersed light-reflective particles with a $25-\mu \mathrm{m}$ diameter: (b) no actuation; (c) vortex rotation caused by exciting one single actuator at $12 \mathrm{MHz}$; (d) particle accumulation on circular lines with both the actuators excited at $8.2 \mathrm{MHz}$ and a phase difference of $0^{\circ}$.

Acknowledgments: The present work is funded by the project "MIcrosystems meRging ACoustics and fLuidics to build human engineered tissuE (MIRACLE)" funded by University of Brescia in the context of "Health and Wealth 2015" athenaeum grant.

Conflicts of Interest: The authors declare no conflict of interest.

\section{References}

1. Fu, Y.Q.; Luo, J.K.; Nguyen, N.T.; Walton, A.J.; Flewitt, A.J.; Zu, X.T.; Li, Y.; McHale, G.; Matthews, A.; Iborra, E.; et al. Advances in piezoelectric thin films for acoustic biosensors, acoustofluidics and lab-on-chip applications. Prog. Mater. Sci. 2017, 89, 31-91, doi:10.1016/j.pmatsci.2017.04.006.

2. Shilton, R.; Tan, M.K.; Yeo, L.Y.; Friend, J.R. Particle concentration and mixing in microdrops driven by focused surface acoustic waves. J. Appl. Phys. 2008, 104, 014910, doi:10.1063/1.2951467.

3. Ding, X.; Lin S.S.; Kiraly, B.; Yue, H., Li; S., Chiang, I.; Shi, J.; Benkovic, S.J.; Huang, T.J. On-chip manipulation of single microparticles, cells, and organisms using surface acoustic waves. PNAS 2012, 109, 11105-11109, doi:10.1073/pnas.1209288109.

4. Orloff, N.D.; Dennis, J.R.; Cecchini, M.; Schonbrun, E.; Rocas, E.; Wang, Y.; Novotny, D.; Simmonds, R.W.; Moreland, J.; Takeuchi, I.; et al. Manipulating particle trajectories with phase-control in surface acoustic wave microfluidics. Biomicrofluidics 2011, 5, 044107, doi:10.1063/1.3661129.

5. Ferrari, V.; Lucklum, R. Overview of Acoustic-Wave Microsensors. In Piezoelectric Transducers and Applications, 2nd ed.; Vives, A.A., Ed.; Springer: Berlin/Heidelberg, Germany, 2008; pp. 39-62. 
6. Ferrari, V.; Marioli, D.; Taroni, A. Theory, modeling and characterization of PZT-on-alumina resonant piezo-layers as acoustic-wave mass sensors. Sens. Actuators A 2000, 92, 182-190, doi:10.1016/S0924-4247(01)00561-1.

7. Royer, D.; Dieulesaint, E. Elastic Waves in Solids I: Free and Guided Propagation; Springer: Berlin/Heidelberg, Germany, 2000.

(C) 2017 by the authors. Licensee MDPI, Basel, Switzerland. This article is an open access article distributed under the terms and conditions of the Creative Commons Attribution (CC BY) license (http://creativecommons.org/licenses/by/4.0/). 